\title{
Price-based adaptive spinning reserve requirements in power system scheduling ${ }^{1}$
}

\author{
Chung-Li Tseng ${ }^{\mathrm{a}}$, Shmuel S. Oren ${ }^{\mathrm{a}}$, Alva J. Svoboda ${ }^{\mathrm{b}}$, Raymond B. Johnson ${ }^{\mathrm{b}}$ \\ ${ }^{a}$ Department of Industrial Engineering and Operations Research, University of California, Berkeley, CA 94720, USA \\ ${ }^{\mathrm{b}}$ Pacific Gas \& Electric Company, San Francisco, CA 94177, USA
}

\begin{abstract}
In a deregulated electricity market such as the California WEPEX, spinning reserves must be explicitly identified as an ancillary service and priced. Additionally, scheduling coordinators who match suppliers and demands may either self-provide spinning reserves, or rely on the Independent System Operator (ISO) to provide reserves at the spot price. The deregulated market structure makes explicit the implicit softness that has always been recognized in the reserve constraints: additional reserves may have value even when a minimum reserve requirement has been met. In this paper we formulate the spinning reserve requirement (SRR) as a function of the endogenously determined marginal values of reserves. The spinning reserve requirement depends, according to a non-increasing response function, on a price/value signal. We present three power system scheduling algorithms in which this price/value signal is updated at each iteration of a dual optimization. Game theory is used to interpret the proposed algorithms. Numerical test results are also presented. (C) 1998 Elsevier Science Ltd. All rights reserved.
\end{abstract}

Keywords: Power system scheduling; Unit commitment; Game theory

\section{Introduction}

The solution of power system scheduling problems is essential to a power utility such as PG\&E for major unit commitment and transaction decisions. Many optimization methods have been proposed to solve this problem [1]. Among them, Lagrangian relaxation methods are now widely used approaches to solve unit commitment [2-4]. At PG\&E, the Hydro-Thermal Optimization (HTO) program was developed almost a decade ago, based on the Lagrangian relaxation approach [3]. In our recent work, the Lagrangian relaxation-based algorithm has been extended to schedule thermal units under ramp rate constraints [5] and transmission constraints [6].

The objective of the unit commitment is to minimize the total generating costs of the power system over the planning horizon subject to system constraints (e.g. load balance constraints and spinning reserve constraints) and unit constraints (e.g. minimum up/down time constraints and ramp constraints). Most of the unit constraints describe the physical limits of generation units and should not be violated.

In contrast to these 'hard' constraints, the spinning

\footnotetext{
${ }^{1}$ The authors remain solely responsible for the errors in this paper. This paper does not represent the views of PG\&E or its members.
}

reserve constraints are 'soft', both in the sense that they do not represent actual physical limits (normally they are determined by regulation or law), and in the sense that there may be value-added in exceeding the set level. A deregulated electricity market structure makes explicit the implicit softness that has always been recognized in the reserve constraints. In the deregulated electricity market proposed in California, spinning reserve has been explicitly identified as an ancillary service that will be priced. Additionally, coordinators who match suppliers and demands (in California, for example, the Power Exchange, which will initially be responsible for matching most current utility-owned generation and load) may either self-provide spinning reserves or rely on the independent system operator (ISO), who conducts an auction to provide a spot price for reserves.

The softness of the reserve constraints has previously been dealt with using fuzzy logic techniques [7]. In Ref. [7], Guan et al. proposed an efficient fuzzy optimizationbased method to solve the unit commitment problem with soft reserve requirements. They first convert the problem to a 'crisp' one, then take advantage of separability of the problem and solve it by Lagrangian relaxation. They also demonstrate the trade-off between cost minimization and system reserve satisfaction. However, to avoid infeasibility, the aspiration level of the generation cost is obtained by running the crisp problem with the lowest acceptable 
reserve requirement. This requires multiple runs of the unit commitment algorithm.

Hourly spinning reserve requirements are usually defined to be the greater of a fixed percentage of total forecast demand and the largest on-line unit. A utility or scheduling coordinator may however wish to increase the SRR to a higher level, provided the additional reserves do not cost too much. In this situation, the unit commitment program as usually formulated would probably first be run with the higher SRR. If the marginal cost of reserves at that level were too high, or no feasible commitment satisfying the requirement could be found, the operator would run the program again with a lower requirement, though still greater than or equal to the minimum reserve requirement defined by statute or scheduling protocol. Such a trial and error approach requires multiple runs of the unit commitment program and is time-consuming.

In this paper, we propose a new approach embedded in the Lagrangian relaxation approach. The SRR is defined as adaptive and adjustable between two levels of reserve requirement in all hours. The SRR is adjusted based on the corresponding Lagrange multiplier, which is viewed as price information. Game theory is used to interpret our proposed algorithms. The method proposed in this paper does not require the multiple runs of unit commitment required by other approaches.

We present a formulation of the thermal unit commitment problem in Section 2. In Section 3, after a brief review of the Lagrangian relaxation formulation, we interpret this formulation by a two-firm model, from which the adaptive pricebased SRR is derived. A three-phase algorithm is then presented in Section 4 to solve the unit commitment problem with the adaptive SRR. Numerical test results and conclusions are given in Sections 5 and 6.

\section{Problem formulation}

In this paper the following standard notation will be used. Additional symbols will be introduced when necessary. $i:$

$t:$ $u_{i t}$ :

$x_{i t}$ : state variable indicating the length of time that unit $i$ has been up or down in time period $t$

$t_{i}^{\text {on }}: \quad$ the minimum number of periods unit $i$

must remain on after it has been turned on

$t_{i}^{\text {off }}$ : the minimum number of periods unit $i$ must remain off after it has been turned off

$p_{i t}$ : $\quad$ state variable indicating the amount of

$p_{i}^{\min }: \quad \quad$ minimum rated capacity of unit $i$

$p_{i}^{\max }$ : $\quad$ maximum rated capacity of unit $i$ $r_{i}^{\max }: \quad \quad$ maximum reserve for unit $i$

$r_{i}\left(p_{i t}\right): \quad \quad$ reserve available from unit $i$ in time period $t\left(=\min \left(r_{i}^{\max }, p_{i}^{\max }-p_{i t}\right)\right)$

$C_{i}\left(p_{i t}\right)$ fuel cost for operating unit $i$ at output level $p_{i t}$ in time period $t$

$S_{i}\left(x_{i, t-1}, u_{i t}, u_{i, t-1}\right)$ : start-up cost associated with turning on unit $i$ at the beginning of time period $t$

$D_{t}: \quad$ forecast demand requirement in time period $t$

$R_{t}$ : $\quad$ spinning reserve requirement (SRR) in time period $t$

The unit commitment problem is formulated as the following mixed-integer programming problem (note that the underlined variables are vectors in this paper, e.g. $\left.\underline{u}=\left(u_{11}, \cdots, u_{I T}\right)\right)$

(P) $\min _{\underline{u}, \underline{x}, \underline{p}, \underline{t}} \sum_{t=1}^{T} \sum_{i=1}^{I}\left[C_{i}\left(p_{i t}\right) u_{i t}+S_{i}\left(x_{i, t-1}, u_{i t}, u_{i, t-1}\right)\right]$

subject to the demand constraints,

$\sum_{i=1}^{I} p_{i t} u_{i t}=D_{t}, t=1, \cdots, T$

and the spinning reserve constraints,

$\sum_{i=1}^{I} r_{i}\left(p_{i t}\right) u_{i t} \geq R_{t}, t=1, \cdots, T$

There are other unit constraints such as unit capacity constraints,

$p_{i}^{\min } \leq p_{i t} \leq p_{i}^{\max }, i=1, \cdots, I ; t=1, \cdots, T$

the state transition equation for $i=1, \cdots, I$,

$x_{i t}= \begin{cases}\max \left(x_{i, t-1}, 0\right)+1, & \text { if } u_{i t}=1 \\ \min \left(x_{i, t-1}, 0\right)-1, & \text { if } u_{i t}=0\end{cases}$

the minimum up/down time constraints for $i=1, \cdots, I$,

$u_{i t}=\left\{\begin{array}{cc}1, & \text { if } 1 \leq x_{i, t-1}<t_{i}^{\text {on }} \\ 0, & \text { if }-1 \geq x_{i, t-1}>-t_{i}^{\text {off }} \\ 0 \text { or } 1, & \text { otherwise }\end{array}\right.$

and the initial conditions on $x_{i t}$ at $t=0$ for $\forall i$.

\section{Two-firm model}

The method proposed in this paper is embedded in the Lagrangian relaxation approach. In this section, we will use a two-firm model to interpret this approach. The Lagrangian relaxation approach relaxes the demand constraints and the spinning reserve constraints by using Lagrange multipliers. The problem is then decomposed into $I$ subproblems. Let $\lambda_{t}$ and $\mu_{t}(t=1, \cdots, T)$ be the corresponding non-negative Lagrange multipliers to Eqs. (2) and (3). We have the 


$$
\max _{\underline{\lambda}, \underline{\mu} \geq 0} \min _{\underline{u}, \underline{x}, \underline{p}}\left(\begin{array}{c}
\sum_{t=1}^{T} \sum_{i=1}^{I}\left[C_{i}\left(p_{i t}\right) u_{i t}+S_{i}\left(x_{i, t-1}, u_{i t}, u_{i, t-1}\right)\right]+ \\
\sum_{t=1}^{T}\left[\lambda_{t}\left(D_{t}-\sum_{i=1}^{I} p_{i t} u_{i t}\right)+\mu_{t}\left(R_{t}-\sum_{i=1}^{I} r_{i}\left(p_{i t}\right) u_{i t}\right)\right]
\end{array}\right)
$$

Firm P's cost minimization problem

Firm Q's revenue maximization problem

Fig. 1. The two-firm model.

following dual problem:

$$
\text { (D) } \max _{\underline{\lambda}, \underline{\mu} \geq 0} d(\underline{\lambda}, \underline{\mu} ; \underline{R})
$$

where

$$
d(\underline{\lambda}, \underline{\mu} ; \underline{R})=\sum_{i=1}^{I} d_{i}(\underline{\lambda}, \underline{\mu})+\sum_{t=1}^{T}\left(\lambda_{t} D_{t}+\mu_{t} R_{t}\right)
$$

and

$$
\begin{aligned}
d_{i}(\underline{\lambda}, \underline{\mu})= & \min _{\underline{u}, \underline{x}, \underline{p} \underline{\underline{n}}} \sum_{t=1}^{T}\left[C_{i}\left(p_{i t}\right) u_{i t}+S_{i}\left(x_{i, t-1}, u_{i t}, u_{i, t-1}\right)\right. \\
& \left.-\lambda_{t} p_{i t} u_{i t}-\mu_{t} r_{i}\left(p_{i t}\right) u_{i t}\right]
\end{aligned}
$$

\subsection{Two-firm model with fixed SRR}

Each unit subproblem $d_{i}$ can be interpreted as a profit maximization problem for unit $i[8,9]$, where unit $i$ is an endogenously priced resource and $\lambda_{t}$ and $\mu_{t}$ are the prices paid to the resource.

We interpret the dual optimization using the following two-firm model: Firm $\mathrm{P}$, a power utility, facing demand $D_{t}$ needs to purchase fuel from firm $\mathrm{Q}$ for generation. Fuel for different generating units may vary and fuel cost is described by $C_{i}(\cdot)$. Firm $\mathrm{Q}$ also sells power and spinning reserves. It offers firm $\mathrm{P}$ the prices $\lambda_{t}$ and $\mu_{t}$ for power and spinning reserves at hour $t$ respectively. Firm P's objective is to minimize its total cost given two options: buy fuel and self-generate or directly buy power from firm Q. Firm Q's problem is to adjust the prices of $\lambda_{t}$ and $\mu_{t}$ so as to achieve its maximum revenue, considering that customer firm $\mathrm{P}$ will minimize its cost.

\section{Remarks:}

1. In Fig. 1 the dual objective function is divided into two optimization problems of two firms. If firm $\mathrm{P}$ decides to purchase fuel, its spending is captured by the two terms in the first bracket in the objective function. If firm $\mathrm{P}$ purchases either power or spinning capacity from firm $\mathrm{Q}$, it pays the corresponding amounts in the second bracket. No matter what option firm P chooses, firm Q collects the money.

2. At iteration $k$, firm $\mathrm{Q}$ offers $\lambda_{t}^{k}$ and $\mu_{t}^{k}$, and firm $\mathrm{P}$ obtains $p_{i t}^{k}, u_{i t}^{k}$. If the subgradient $\left(D_{t}-\sum_{i=1}^{I} p_{i t}^{k} u_{i t}^{k}\right)>0, \lambda_{t}^{k}$ should be increased at the next iteration. From firm Q's perspective if $\left(D_{t}-\sum_{i=1}^{I} p_{i t}^{k} u_{i t}^{k}\right)>0$ there is an excess demand for power, and firm $\mathrm{Q}$ could raise the price.

3. Raising $\lambda_{t}$ would stimulate the system to increase total generation at hour $t$ and vice versa. Increasing $\mu_{t}$ would at some point make more units turn on. Changing either $\lambda_{t}$ or $\mu_{t}$ would affect spinning reserves, but it can be shown that the amount of spinning reserves only depends on $\lambda_{t}-\mu_{t}$.

This two-firm model can be treated as a 2-player game. Consider a sequential bargaining game of complete and perfect information in which firm $\mathrm{Q}$ (player 1) moves first and decides on prices $\lambda_{t}$ and $\mu_{t}(t=1, \cdots, T)$; firm $\mathrm{P}$ (player 2) observes $\lambda_{t}$ and $\mu_{t}(t=1, \cdots, T)$, and then chooses its optimal generating policy, $\left(u_{i t}, x_{i t}, p_{i t}\right), \forall i, t$. We have the following proposition.

Proposition 1 If $\left(\underline{\lambda}^{*}, \mu^{*} ; \underline{u}^{*}, p^{*}\right)$ is the solution to $(D)$, it is a Nash equilibrium (Ref. [10]) of the above game.

Proof. The solution to $(D)$ is a Nash equilibrium because given the other player's strategy, each player has no incentive to deviate: given firm P's strategy $\left(\underline{u}^{*}, p^{*}\right)$, firm $\mathrm{Q}$ has no incentive to deviate from $\left(\underline{\lambda}^{*}, \mu^{*}\right)$, because these prices maximize its revenue $d$ which is a concave function. Similarly, given firm Q's strategy $\left(\underline{\lambda}^{*}, \mu^{*}\right),\left(\underline{u}^{*}, \underline{p}^{*}\right)$ is the best strategy for firm $\mathrm{P}$, because $\left(\underline{u}^{*}, \underline{p}^{*}\right)$ minimizes $d\left(\underline{\lambda}^{*}, \underline{\mu}^{*}\right)$.

Note that the result of Proposition 1 holds if the game is a simultaneous-move game. However, a sequential game with no need to assume that firm $\mathrm{P}$, a spinning reserve provider, has market power, fits reality better than the model of a simultaneous-move game. The subgradient algorithm therefore can be regarded as an interpretation of how each firm adjusts its decision in the face of the other firm's choice. The sequences $\left\{\left(\underline{\lambda}^{k}, \underline{\mu}^{k}\right)\right\}$ and $\left\{\left(\underline{u}^{k}, \underline{p}^{k}\right)\right\}$, describe a process of adjustment to equilibrium.

\subsection{Price-based adaptive SRR}

In a deregulated electricity market such as the one proposed in California, spinning reserve is obtained and priced as an ancillary service. The ISO has ultimate responsibility for meeting the reserve requirements based on predetermined operating guidelines. Scheduling 


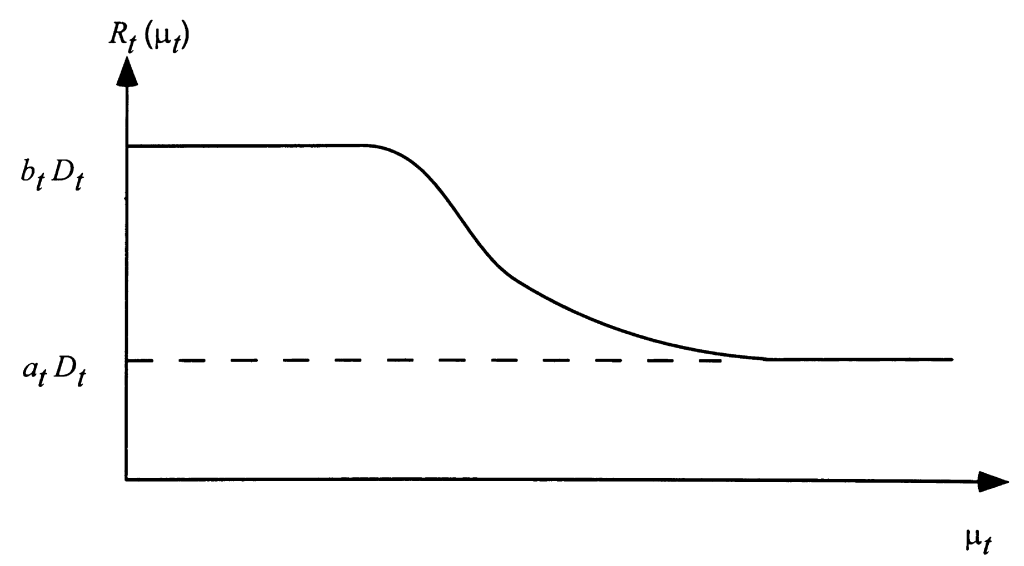

Fig. 2. An example of $R_{t}\left(\mu_{t}\right)$.

coordinators have the option to provide some or all of their reserve requirements. If they do not self-provide, the ISO will do so at the spot price. A coordinator thus faces the problems of estimating the value (or opportunity cost) of reserves, and then of deciding on its levels of self-provision of reserves, and whether to bid into the reserve auction, based on this value. In this paper, we propose a scheme for obtaining an optimal level of spinning reserves at or above a minimum requirement, on the assumption that additional reserves have value in at least some hours. The flexible SRR avoids uneconomic solutions in which expensive units are unnecessarily committed, and also avoids cases in which requiring desired higher levels of reserves would result in the program not finding a feasible unit commitment. These phenomena both tend to be indicated by high values of the multipliers on spinning reserves. A flexible SRR which is formulated as a function of an iteratively determined value of reserves can therefore also be used to improve trade-offs between optimality and feasibility in the unit commitment solution.

We now modify the two-firm model defined in Section 3.1. Let firm $P$ now also select its SRR. Firm Q, as in the original model, offers prices $\underline{\lambda}$ and $\mu$. After observing the prices $\underline{\lambda}$ and $\underline{\mu}$, firm P defines its SRR first, then solves the corresponding optimal generating policy, $\left\{u_{i t}, p_{i t}\right\}$ for all $i, t$. We assume that $R_{t}$ is selected based on a function of $\mu_{t}$ in the form $\theta\left(\mu_{t}\right) D_{t}$, where $\theta: \Re^{+} \rightarrow\left[a_{t}, b_{t}\right]$ is a monotone nonincreasing function. For simplicity, we denote it as $R_{t}\left(\mu_{t}\right)$. $R_{t}(\cdot)$ is called the response function of firm $\mathrm{P}$. This means that when $\mu_{t}$, the price of spinning capacity offered by $\mathrm{Q}$, is high, firm P not only tends to commit more units (as in the original model) but also wants to lower its SRR, and vice versa. An example of $R_{t}\left(\mu_{t}\right)$ is depicted in Fig. 2, where $R_{t}\left(\mu_{t}\right)=\left[\left(b_{t}+a_{t}\right) / 2+\left(b_{t}-a_{t}\right) / 2 \cdot \tanh \left(-\beta\left(\mu_{t}-\alpha\right)\right)\right]$ $D_{t}, \alpha>0, \beta>0$.

In this extended two-firm model, the SRR is driven by price through a response function. This corresponds to a variation of the dual problem $(D)$. We will present the formulation along with the solution procedure in the following section.

\section{The solution procedure}

Despite the efficiency of solving $(D)$ due to its separability, the solution of $(D)$ does not necessarily yield a feasible solution to $(P)$, i.e. satisfying Eqs. (2) and (3). Lagrangian relaxation algorithms for solving unit commitment have often been implemented with two phases: a dual optimization phase and a feasibility phase. In Ref. [11], a three-phase structure of an algorithm for solving unit commitment problems is proposed as follows.

Phase 1: Dual optimization, i.e. solving $(D)$.

Phase 2: Feasibility phase: to obtain a feasible schedule. Phase 3: Unit decommitment phase.

In the following sections, we will first briefly review the three-phase algorithm for solving the unit commitment with fixed SRR. With little modification, the three-phase algorithm is extended to handle the case with flexible SRR.

\subsection{Three-phase algorithm with fixed SRR}

It is well known that the dual objective function $d$ is concave and continuous but not necessarily differentiable at all points. A subgradient algorithm is applied to solve the dual problem $(D)$. It can be shown that the vector of the mismatches in the demand constraints and the spinning reserve constraints is a subgradient of the dual objective function $d$ [12].

\section{Phase 1: The subgradient algorithm}

Step 0: $k \leftarrow 0 ; \underline{\lambda}^{0}$ and $\underline{\mu}^{0}$ are given.

Step 1: If stopping criteria are met, stop. Otherwise solve $d\left(\underline{\lambda}^{k}, \underline{\mu}^{k} ; \underline{R}\right)$ to obtain $\left(\underline{u}^{k}, \underline{p}^{k}\right)$.

Step 2: $\lambda_{t}^{k+1}=\lambda_{t}^{k}+s^{k}\left(D_{t}-\sum_{i=1}^{I} p_{i t}^{k} u_{i t}^{k}\right), \mu_{t}^{k+1}=\max \left(0, \mu_{t}^{k}\right.$ $\left.+s^{k}\left(R_{t}-\sum_{i=1}^{I} r_{i}\left(p_{i t}\right) u_{i t}^{k}\right)\right), \forall t$.

Step 3: $k \leftarrow k+1$, go to Step 1 . 
In Ref. [13], it is shown that under some conditions on the step size $s_{k}$, the subgradient algorithm converges. The stopping criteria used in our implementation include the maximum number of iterations, the change of norm of subgradients at two consecutive iterations and the number of iterations without improvement in the dual objective value.

In the Phase 2 proposed in Ref. [14], the hour of the mostviolated SRR is determined, and the corresponding $\mu_{t}$ is enhanced. A method is proposed to calculate the exact amount of the increase in the value of the corresponding $\mu_{t}$ to satisfy SRR at the corresponding hour. However, this method seems to take a long time to locate a feasible solution because only one $\mu_{t}$ is updated at a time. A natural extension is to simultaneously update at each iteration the $\mu_{t}$ corresponding to all the hours that the SRR is violated [3,2]. This speeds up the feasibility phase at a cost of possible overcommitment in the generating units [2]. Under the structure of the three-phase algorithm, this overcommitment can be corrected in the unit decommitment phase to be discussed later. Although these Phase II methods were all designed for the case where spinning capacity constraints are of the form below:

$\sum_{i=1}^{I} p_{i}^{\max } u_{i t} \geq D_{t}+R_{t}, \forall t$

the basic idea behind them can actually be applied to the reserve constraints of the form in Eq. (3).

The purpose of a Phase 2 algorithm is to obtain a feasible solution, or equivalently, a dispatchable commitment. We first examine the conditions of existence of a dispatchable commitment. Given a commitment $\left\{\tilde{u}_{i t}\right\}$, it is dispatchable if and only if the following conditions hold $\forall t$.

$\sum_{i=1}^{I} p_{i}^{\min } \tilde{u}_{i t} \leq D_{t} \leq \sum_{i=1}^{I} p_{i}^{\max } \tilde{u}_{i t}$

$\sum_{i=1}^{I} r_{i}^{\max } \tilde{u}_{i t} \geq R_{t}$

and

$\sum_{i=1}^{I} p_{i}^{\max } \tilde{u}_{i t} \geq D_{t}+R_{t}$

The proof of the existence conditions above is straightforward and is omitted here. It can be seen that the existence of a solution requires the satisfaction of sufficiency of both (system) spinning capability, Eqs. (11a) and (11b), and reserve capability Eq. (11c). We now introduce our Phase 2 algorithm.

\section{Phase 2: Feasibility algorithm}

Step 0: $k \leftarrow 0 ; \underline{\lambda}^{0}$ and $\underline{\mu}^{0}$ are from Phase 1 .

Step 1: Given $\underline{\lambda}^{k}$ and $\underline{\mu}^{k}$, solve $d\left(\underline{\lambda}^{k}, \underline{\mu}^{k} ; \underline{R}\right)$ to obtain $\left(\underline{u}^{k}, \underline{p}^{k}\right)$.
Step 2: If the existence conditions Eqs. (11a), (11b) and (11c), applied to $\left\{u_{i t}^{k}\right\}$, are satisfied, stop and $\left\{u_{i t}^{k}\right\}$ is dispatchable.

Step 3: $\quad \lambda_{t}^{k+1}=\lambda_{t}^{k}, \mu_{t}^{k+1}=\mu_{t}^{k}+s^{k} \cdot \Delta \mu_{t}^{k}, \Delta \mu_{t}^{k}>0 \forall t$ violating the existence conditions Eqs. (11a), (11b) and (11c), applied to $\left\{u_{i t}^{k}\right\}$.

Step 4: $k \leftarrow k+1$, go to Step 1 .

In Step 2 of the algorithm above, for the hours with insufficient (system) spinning or reserve capability, the corresponding $\mu_{t}$ are enhanced by $\Delta \mu_{t}^{k}$. This, based on our two-firm interpretation, will induce more unit commitment.

A unit decommitment method has been developed as a postprocessing phase (Phase 3) for the algorithms for solving the unit commitment problem. Given a feasible unit commitment $(\underline{\tilde{u}}, \tilde{p})$, the unit decommitment method improves the solution while maintaining feasibility. In Ref. [15] it is shown that the unit decommitment method not only improves solution quality generally, but also mitigates unpredictable effects due to heuristics in the first two phases. The interested reader can refer to Refs $[11,15]$ for detailed discussion.

\subsection{Three-phase algorithm with adaptive $S R R$}

In Section 3.2, the concept of price-based adaptive SRR was introduced. This flexible SRR of firm $\mathrm{P}$ is captured by a response function $R_{t}(\cdot)$ at time $t$. Depending on whether firm $\mathrm{Q}$ knows the response function of firm $\mathrm{P}$, firm Q's decision for price adjustment varies. This creates two variations of the dual problem $(D)$, Nash-type and Stackelberg-type twofirm models, to be discussed next.

\subsubsection{Sequential bargaining game model}

Consider a sequential bargaining game of complete and perfect information in which firm $\mathrm{Q}$ moves first and firm $\mathrm{P}$ moves second. The timing of the game is as follows: (1) firm $\mathrm{Q}$ chooses the prices $\lambda_{t}$ and $\mu_{t}$, for all $t$; (2) firm $\mathrm{P}$ observes $\lambda_{t}$ and $\mu_{t}$, and then chooses its SRR and the corresponding optimal generating policy, $u_{i t}$ and $p_{i t}$. Assume the scenario is common knowledge to both firms $\mathrm{P}$ and $\mathrm{Q}$, but firm $\mathrm{Q}$ does not know the response function of firm P. In the following, we use the notation $\underline{R}\left(\underline{\mu)}=\left(R_{1}\left(\mu_{1}\right), \ldots, R_{T}\left(\mu_{T}\right)\right)\right.$.

Proposition 2 If $\left(\underline{\lambda}^{*}, \mu^{*} ; \underline{u}^{*}, p^{*}\right)$ is a solution to the following problem $\left(D^{*}\right)$, then $\left(\underline{\lambda}^{*}, \mu^{*} ; \underline{R}\left(\mu^{*}\right), \underline{u}^{*}, p^{*}\right)$ is a Nash equilibrium of the sequential bargaining game.

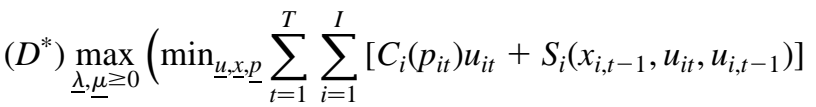

$$
\begin{aligned}
& +\sum_{t=1}^{T}\left[\lambda_{t}\left(D_{t}-\sum_{i=1}^{I} p_{i t} u_{i t}\right)+\mu_{t}\left(R_{t}^{*}-\sum_{i=1}^{I} r_{i}\left(p_{i t}\right) u_{i t}\right)\right]
\end{aligned}
$$$$
\left.\times \text { subject to } R_{t}^{*}=R_{t}\left(\mu_{t}^{*}\right)\right)
$$ 
To find a Nash equilibrium, we construct an algorithm to simulate the process of adjustment toward equilibrium. At each iteration, as in the subgradient algorithm, firm Q adjusts prices based on the law of supply and firm P minimizes its cost. Also firm P updates its SRR in response to firm Q's price, i.e. $R_{t}^{k+1}=R_{t}\left(\mu_{t}^{k}\right)$. This means that in order to achieve $R_{t}^{*}=R_{t}\left(\mu_{t}^{*}\right)$, firm P approximates $\mu_{t}^{*}$ by $\mu_{t}^{k}$.

\section{Nash-type Phase 1 Algorithm}

Step 0: $\quad k \leftarrow 0 ;\left\{\lambda_{t}^{0}\right\}$ and $\left\{\mu_{t}^{0}\right\}$ are given.

Step 1: Given $\underline{\lambda}^{k}, \mu^{k}$, solve $d\left(\underline{\lambda}^{k}, \mu^{k} ; \underline{R}\left(\mu^{k}\right)\right)$ to obtain $\left(\underline{u}^{k}, p^{k}\right)$.

Step 2: $\quad \lambda_{t}^{k+1}=\lambda_{t}^{k}+s^{k}\left(D_{t}-\sum_{i=1}^{I} p_{i t}^{k} u_{i t}^{k}\right), \mu_{t}^{k+1}=$ $\max \left(0, \mu_{t}^{k}+s^{k}\left(R_{t}-\sum_{i=1}^{I} r_{i}\left(p_{i t}^{k}\right) u_{i t}^{k}\right)\right), \forall t$.

Step 3: $\quad k \leftarrow k+1$, go to Step 1 .

The convergence of the Nash algorithm has not yet been established theoretically, but it converges in all observed cases.

\subsubsection{Stackelberg game}

As in the sequential bargaining game defined in Section 4.2 we now consider the case in which firm $Q$ knows the response function of firm $\mathrm{P}$ so that firm $\mathrm{Q}$ expects firm P's response in its revenue maximization problem. This game is commonly known as a Stackelberg game [10].

Proposition 3 If $(\underline{\hat{\lambda}}, \underline{\hat{\mu}} ; \underline{\hat{u}}, \hat{p})$ is a solution to the following problem $(\hat{D})$, then $(\underline{\hat{\lambda}}, \underline{\hat{\mu}} ; \underline{R}(\underline{\hat{u}}), \underline{\hat{u}}, \underline{\hat{p}})$ is a Stackelberg equilibrium of the Stackelberg game.

$$
\begin{aligned}
& (\hat{D}) \max _{\underline{\lambda}, \underline{\mu} \geq 0} \min _{\underline{u}, \underline{\underline{x}} \underline{\underline{p}} \underline{\underline{.}}}\left(\sum_{t=1}^{T} \sum_{i=1}^{I}\left[C_{i}\left(p_{i t}\right) u_{i t}+S_{i}\left(x_{i, t-1}, u_{i t}, u_{i, t-1}\right)\right]\right. \\
& \left.+\sum_{t=1}^{T}\left[\lambda_{t}\left(D_{t}-\sum_{i=1}^{I} p_{i t} u_{i t}\right)+\mu_{t}\left(R_{t}\left(\mu_{t}\right)-\sum_{i=1}^{I} r_{i}\left(p_{i t}\right) u_{i t}\right)\right]\right)
\end{aligned}
$$

We assume that $R_{t}(\cdot)$ is continuously differentiable for all $t$. We can then apply the subgradient algorithm to solve $(\hat{D})$ to obtain a Stackelberg equilibrium.

\section{Stackelberg-type Phase 1 Algorithm}

Step 0: $\quad k \leftarrow 0 ; \underline{\lambda}^{0}$ and $\underline{\mu}^{0}$ are given.

Step 1: Given $\underline{\lambda}^{k}$ and $\mu^{k}$, solve $d\left(\underline{\lambda}^{k}, \mu^{k} ; \underline{R}\left(\mu^{k}\right)\right)$ to obtain $\left(\underline{u}^{k}, p^{k}\right) \forall i, t$.

Step 2: $\quad \lambda_{t}^{k+1}=\lambda_{t}^{k}+s^{k}\left(D_{t}-\sum_{i=1}^{I} p_{i t}^{k} u_{i t}^{k}\right), \mu_{t}^{k+1}=$ $\max \left(0, \mu_{t}^{k}+s^{k}\left(R_{t}\left(\mu_{t}^{k}\right)-\sum_{i=1}^{I} r_{i}\left(p_{i t}^{k}\right) u_{i t}^{k}\right.\right.$ $\left.\left.+\mu^{k_{t}} R_{t}^{\prime}\left(\mu_{t}^{k}\right)\right)\right), \forall t$.

Step 3: $\quad k \leftarrow k+1$, go to Step 1 .
(Note that the objective of $(\hat{D})$ is not necessarily concave because $\sum_{t=1}^{T} \mu_{t} R_{t}{ }^{\prime}\left(\mu_{t}\right)$ may not be concave.)

The feasibility algorithm for the case with flexible SRR is basically the same as the Phase 2 algorithm presented in Section 4.1 with the fixed SRR $R_{t}$ replaced by the flexible one $R_{t}(\cdot)$, as a function of appropriate $\mu_{t}$. For simplicity, we will not restate the Phase 2 algorithm here. After Phase 2, a feasible schedule corresponding to an SRR will be obtained. The SRR determined from the final iteration of Phase 2 can therefore be regarded as the system SRR. A unit decommitment method can then be applied to improve the solution quality with this system SRR fixed.

\subsection{Discussion}

Let $L$ be the Lagrangian of the unit commitment problem $(P)$ :

$$
\begin{gathered}
L(\underline{x}, \underline{u}, \underline{p} ; \underline{\lambda}, \underline{\mu}, \underline{R}) \equiv \sum_{t=1}^{T} \sum_{i=1}^{I}\left[C_{i}\left(p_{i t}\right) u_{i t}+S_{i}\left(x_{i, t-1}, u_{i t}, u_{i, t-1}\right)\right] \\
+\sum_{t=1}^{T}\left[\lambda_{t}\left(D_{t}-\sum_{i=1}^{I} p_{i t} u_{i t}\right)+\mu_{t}\left(R_{t}-\sum_{i=1}^{I} r_{i}\left(p_{i t}\right) u_{i t}\right)\right]
\end{gathered}
$$

Suppose $0.05 D_{t}=R_{t}^{\min } \leq R_{t}\left(\mu_{t}\right) \leq R_{t}^{\max }=0.07 D_{t}$ for all $\mu_{t} \geq 0, \forall_{t}$, and $\left(\underline{\lambda}^{*}, \mu^{*}\right)$ and $(\underline{\hat{\lambda}}, \hat{\mu})$ solve $\left(D^{*}\right)$ and $(\hat{D})$ respectively. We have

$$
\begin{aligned}
& \max _{\underline{\lambda}, \underline{\mu} \geq 0} \min _{\underline{x}, \underline{u}, \underline{p}} L\left(\underline{x}, \underline{u}, \underline{p} ; \underline{\lambda}, \underline{\mu}, \underline{R}^{\min }\right) \\
& \leq\left(D^{*}\right) \max _{\underline{\lambda}, \underline{\mu} \geq 0} \min _{\underline{x}, \underline{u}, \underline{p}} L\left(\underline{x}, \underline{u}, \underline{p} ; \underline{\lambda}, \underline{\mu}, \underline{R}\left(\underline{\mu}{ }^{*}\right)\right) \\
& \leq(\hat{D}) \max _{\underline{\lambda}, \underline{\mu} \geq 0} \min _{\underline{x}, \underline{u}, \underline{p}} L(\underline{x}, \underline{u}, \underline{p} ; \underline{\lambda}, \underline{\mu}, \underline{R}(\underline{\mu})) \\
& \leq \max _{\underline{\lambda}, \underline{\mu} \geq 0} \min _{\underline{x}, \underline{u}, \underline{p}} L(\underline{x}, \underline{u}, \underline{p} ; \underline{\lambda}, \underline{\mu}, \underline{R}(\underline{\hat{\mu}})) \\
& \leq \max _{\underline{\lambda}, \underline{\mu} \geq 0} \min _{\underline{x}, \underline{u}, \underline{p}} L\left(\underline{x}, \underline{u}, \underline{p} ; \underline{\lambda}, \underline{\mu}, \underline{R}^{\max }\right)
\end{aligned}
$$

To see the inequality between Eqs. (16) and (17), note that the optimal solution $\left(\underline{\lambda}^{*}, \mu^{*}\right)$ of $\left(D^{*}\right)$ is feasible to $(\hat{D})$. This shows that the revenue of the leader (firm Q) in the Stackelberg game exceeds that in the sequential bargaining game defined in Section 4.2. This is because in the Stackelberg game firm $\mathrm{Q}$ has more information about firm $\mathrm{P}$ than in the sequential bargaining game.

Both the Nash algorithm and the Stackelberg algorithm can be applied to obtain a price-based adaptive SRR. For example, if we solve $(\hat{D})$ and obtain $(\underline{\hat{\lambda}}, \underline{\hat{\mu}})$, we can use $\underline{R}(\hat{\mu})$ as SRR to define a unit commitment $(\bar{P})$ as below.

$$
\begin{aligned}
& (\bar{P}) \min _{\underline{u}, \underline{x}, \underline{\underline{p}}} \sum_{t=1}^{T} \sum_{i=1}^{I}\left[C_{i}\left(p_{i t}\right) u_{i t}+S_{i}\left(x_{i, t-1}, u_{i t}, u_{i, t-1}\right)\right] \\
& \text { s.t. } \sum_{i=1}^{I} p_{i t} u_{i t}=D_{t} ; \sum_{i=1}^{I} r_{i}\left(p_{i t}\right) u_{i t} R_{t}\left(\hat{\mu}_{t}\right), \forall t
\end{aligned}
$$


Table 1

Comparison of methods

\begin{tabular}{|c|c|c|c|c|c|}
\hline \multirow[t]{2}{*}{ Methods } & \multirow[t]{2}{*}{ Total cost $(\$)$} & \multicolumn{3}{|c|}{ CPU time (s) } & \multirow[t]{2}{*}{ Duality gap (\%) } \\
\hline & & Phase 1 & Phase 2 & Phase 3 & \\
\hline Fixed SRR* & 9333747 & 11.53 & 10.1 & 0.13 & 0.70 \\
\hline Adaptive SRR1† & 9296353 & 10.17 & 7.64 & 0.29 & 0.40 \\
\hline Adaptive SRR2末 & 9291727 & 11.56 & 7.22 & 0.12 & 0.35 \\
\hline
\end{tabular}

* Fixed SRR: $7 \% \times$ load.

$\dagger$ Adaptive SRR1: Nash algorithm in Phase 1.

† Adaptive SRR2: Stackelberg algorithm in Phase 1 .

The corresponding dual problem $(\hat{D})$ of $(\hat{P})$ is as follows.

(D) $\max _{\underline{\lambda}, \mu \geq 0} \min _{\underline{x}, \underline{u}, \underline{p}} L(\underline{x}, \underline{u}, \underline{p} ; \underline{\lambda}, \underline{\mu}, \underline{R}(\underline{\hat{\mu}}))$

Without really solving $(\bar{D})$, we approximate the duality gap between $(\bar{P})$ and $(\bar{D})$ by the 'duality' gap between $(\bar{P})$ and $(\hat{D})$. Note that this approximation is more restrictive (with a larger value) than the value of the actual duality gap. In our experiments, the 'duality' gap between $(\hat{D})$ and $(\bar{P})$ is within $1.5 \%$ for 10 -unit-168-hour cases, and within $0.3 \%$ for 30 unit-168-hour cases. The real duality gaps between $(\bar{P}) \wedge(\bar{D})$ for both cases are actually smaller.

\section{Numerical results}

The algorithms are implemented in FORTRAN on an HP 700 workstation. A 30-unit thermal model problem over a one week planning horizon is tested. The total system capacity is $15515 \mathrm{MW}$ which is much higher than the peak load. We apply the three-phase algorithm to solve the unit commitment problem. In this test problem, we let the interval of SSR $\left[a_{t} D_{t}, b_{t} D_{t}\right]$ be $\left[0.05 D_{t}, 0.07 D_{t}\right]$, for all $t$. The conventional fixed SRR method $\left(R_{t}=0.07 D_{t}\right.$, for all $\left.t\right)$ is compared with the adaptive SRR method. We have also tested both Nash and Stackelberg algorithms in Phase 1 in the adaptive SRR case. The response function used in this test problem has the form:

$R_{t}\left(\mu_{t}\right)=0.06+0.01 \tanh \left(-4\left(\mu_{t}-0.5\right)\right), \forall t$

The algorithm performances are summarized in Table 1.

Both adaptive SRR algorithms have lower total generating cost due to the relaxation of SRR, and achieve better solutions in terms of the duality gap. Also the Stackelberg model outperforms the sequential bargaining model (Nash algorithm) in terms of cost saving because of having more information, as explained in Section 4.

The system spinning capacity profiles obtained by fixed SRR and adaptive SRR (Stackelberg model) methods are depicted in Fig. 3, in which only one day is shown. The corresponding data can be found in Tables 2 and 3. In

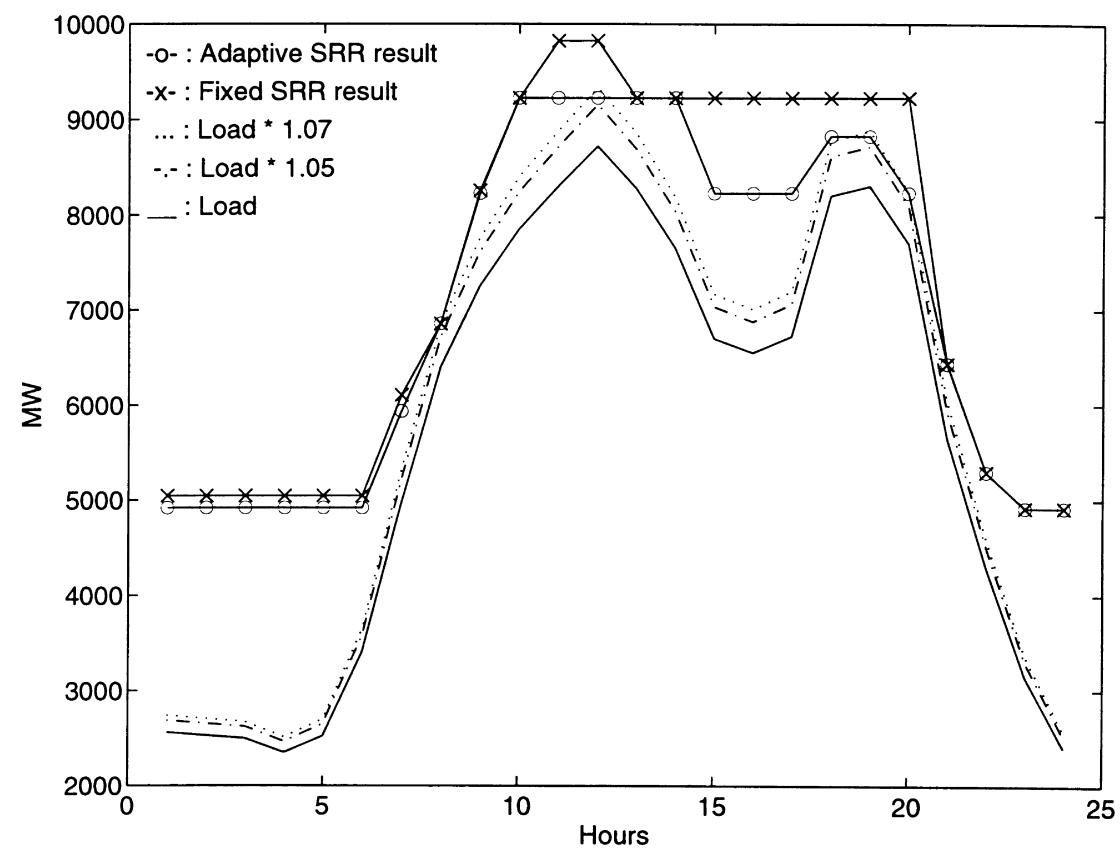

Fig. 3. System spinning capacities and SRR. 
Table 2

$\mu_{t}$ of selected hours (fixed SRR)

\begin{tabular}{|c|c|c|c|c|c|c|c|c|c|c|c|c|}
\hline Hour & 2 & 4 & 6 & 8 & 10 & 12 & 14 & 16 & 18 & 20 & 22 & 24 \\
\hline$\mu_{t}$ & 0 & 0 & 0 & 0.35 & 0.595 & 1.724 & 0.437 & 0 & 0.892 & 0.523 & 0 & 0 \\
\hline SRR (\%) & 7.0 & 7.0 & 7.0 & 7.0 & 7.0 & 7.0 & 7.0 & 7.0 & 7.0 & 7.0 & 7.0 & 7.0 \\
\hline Load (MW) & 2530 & 2350 & 3400 & 6400 & 7850 & 8720 & 7650 & 6550 & 8200 & 7700 & 4300 & 2400 \\
\hline $\mathrm{SSC}(\mathrm{MW}) \dagger$ & 5045 & 5045 & 5045 & 6855 & 9225 & 9825 & 9225 & 9225 & 9225 & 9225 & 5295 & 4920 \\
\hline Reserve $(\%) \ddagger$ & 99.4 & 114.6 & 48.4 & 7.1 & 17.5 & 12.6 & 20.5 & 40.8 & 12.5 & 19.8 & 23.1 & 105 \\
\hline
\end{tabular}

$\uparrow$ SSC: system spinning capacity.

$\ddagger$ Reserve $(\%)=100 \times($ SSC - load $) /$ load.

Table 3

$\mu_{t}$ of selected hours (adaptive SRR)

\begin{tabular}{|c|c|c|c|c|c|c|c|c|c|c|c|c|}
\hline Hour & 2 & 4 & 6 & 8 & 10 & 12 & 14 & 16 & 18 & 20 & 22 & 24 \\
\hline$\mu_{t}$ & 0 & 0 & 0 & 0 & 0.469 & 1.281 & 0.317 & 0 & 0.788 & 0.375 & 0 & 0 \\
\hline SRR (\%) & 7.0 & 7.0 & 7.0 & 7.0 & 6.1 & 5.0 & 6.5 & 7.0 & 5.3 & 6.4 & 7.0 & 7.0 \\
\hline Load (MW) & 2530 & 2350 & 3400 & 6400 & 7850 & 8720 & 7650 & 6550 & 8200 & 7700 & 4300 & 2400 \\
\hline SSC (MW) & 4920 & 4920 & 4920 & 6855 & 9225 & 9225 & 9225 & 8225 & 8825 & 8225 & 5295 & 4920 \\
\hline Reserve (\%) & 94.4 & 109.3 & 44.7 & 7.1 & 17.5 & 5.8 & 20.5 & 25.5 & 7.6 & 6.8 & 23.1 & 105 \\
\hline
\end{tabular}

Table 2 the multipliers $\mu_{t}$ corresponding to fixed (7\%) SRR in selected hours are listed. The value of $\mu_{t}$ in an hour reflects the shadow price of the SRR in the hour. Note that the value of $\mu$ in hour 12 is much higher than that of $\mu_{t}$ in other hours. In Table 3 , the proposed adaptive SRR is applied to the same test problem. It can be seen that the SRR in an hour responds to the corresponding $\mu_{t}$ based on the response function Eq. (21).

In Fig. 3 it can be seen that at hour 12, the SRR has been relaxed down to $5 \%$ of the load and yields an actual reserve of $5.8 \%$ of the load. Slight relaxation of the SRR at hour 12 affects the commitment in the following hours (due to minimum up time or down time constraints). In Fig. 3 it can be seen that during the period between hours 15 and 19, a commitment with adequate but more economic spinning reserve is achieved with adaptive SRR. At the same time higher spinning reserve which increases reliability is maintained when it is inexpensive.

\section{Conclusion}

In this paper we have interpreted the Lagrangian relaxation method for solving the unit commitment as a two-player game: one player chooses unit commitment to minimize cost based on prices; the other adjusts prices to maximize revenue. The optimum solution of the Lagrangian relaxation method is shown to be a Nash equilibrium of this game. We have also shown that the subgradient rule captures the responses of the players. Throughout this interpretation, the game model can include other system parameters as decision variables to the game. For example, 'soft' spinning reserve requirements are made adaptive and respond to a price signal according to a non-increasing response function at each iteration in the dual optimization. The numerical test results show that this method avoids uneconomic unit commitment solutions while enhancing system security. This approach can yield both cost savings and improved algorithm performance. In the context of deregulation, we believe that scheduling coordinators such as the Power Exchange who have the option of selfproviding spinning reserves will find an adaptive representation of their reserve requirements to be the appropriate one in finding their most profitable reserve levels.

\section{Acknowledgements}

This work was partly supported by the National Science Foundation under Grant IRI-9120074 and by PG\&E R\&D Department. Their support is greatly appreciated.

\section{References}

[1] Cohen A, Sherkat V. Optimization-based methods for operations scheduling. Proceedings of IEEE 1987;75(12):1574-1591.

[2] Bertsekas DP, Lauer GS, Sandell Jr. NR, Posbergh TA. Optimal shortterm scheduling of large-scale power systems. IEEE Trans Automatic Control 1983;AC-28(1):1-11.

[3] Ferreira LAFM, Andersson T, Imparato CF, Miller TE, Pang CK, Svoboda A, Vojdani AF. Short-term resource scheduling in multiarea hydrothermal power systems. Electrical Power \& Energy Systems 1989;11(3).

[4] Guan X, Luh PB, Yen H, Rogan P. An optimization-based method for unit commitment. Electrical Power \& Energy Systems 1992;14(1):917.

[5] Svoboda AJ, Tseng CL, Li CA, Johnson RB. Short term resource scheduling with ramp constraints. IEEE Trans Power Systems 1997;12(1):77-83. 
[6] Tseng CL, Oren SS, Cheng CS, Li CA, Svoboda AJ, Johnson RB A transmission-constrained unit commitment method in power system scheduling. Proceedings of the Thirty-first Annual Hawaii International Conference on System Sciences, Vol. III, 1998. pp. $71-80$.

[7] Guan X, Luh PB, Prasannan B. Power system scheduling with fuzzy reserve requirements. IEEE Trans Power Systems 1996;11(2):864869.

[8] Svoboda AJ, Simulation of dispatchable demand-side management in electric power system operations planning. PhD thesis, Department of Industrial Engineering and Operations Research, University of California at Berkeley, November 1992.

[9] Svoboda AJ, Oren SS. Integrating price-based resources in short-term scheduling of electric power systems. IEEE Trans Energy Conversion 1994;9(4):760-769.
[10] Gibbons R, Game theory for applied economists. Princeton: Princeton University Press, 1992.

[11] Tseng CL, On power system generation unit commitment problems. $\mathrm{PhD}$ thesis, Department of Industry Engineering and Operations Research, University of California at Berkeley, December 1996.

[12] Bazaraa MS, Shetty CM, Nonlinear programming - theory and algorithms. New York: Wiley, 1979.

[13] Polyak BT. Minimization of unsmooth functionals. USSR Comput Math Math Phys 1969;9:14-29.

[14] Zhuang F, Galiana FD. Towards a more rigorous and practical unit commitment by Lagrangian relaxation. IEEE Trans Power Systems 1988;PWRS-3(2):763-770.

[15] Tseng CL, Oren SS, Svoboda AJ, Johnson RB. A unit decommitment method in power system scheduling. Electrical Power \& Energy Systems 1997;19(6):357-365. 